\title{
Lung microbiome and host immune tone in subjects with idiopathic pulmonary fibrosis treated with inhaled interferon- $\gamma$
}

\author{
Jing Wang (1) 1,2, Melissa Lesko², Michelle H. Badri ${ }^{3}$, Bianca C. Kapoor ${ }^{2}$, \\ Benjamin G. Wu ${ }^{2}$, Yonghua Li ${ }^{2}$, Gerald C. Smaldone ${ }^{4}$, Richard Bonneau ${ }^{3,5,6}$, \\ Zachary D. Kurtz ${ }^{7}$, Rany Condos ${ }^{2}$ and Leopoldo N. Segal ${ }^{2}$
}

\begin{abstract}
Affiliations: 'Division of Pulmonary and Critical Care Medicine, Beijing Chaoyang Hospital, The Capital University of Medicine, Beijing, China. ${ }^{2}$ Division of Pulmonary, Critical Care and Sleep Medicine, New York University School of Medicine, New York, NY, USA. ${ }^{3}$ Dept of Biology, Center for Genomics and Systems Biology, New York University, New York, NY, USA. ${ }^{4}$ Division of Pulmonary, Critical Care and Sleep Medicine, State University of New York at Stony Brook, Stony Brook, NY, USA. ${ }^{5}$ Courant Institute of Mathematical Sciences, New York University, New York, NY, USA. 'Simons Center for Data Analysis, Simons Foundation, New York, NY, USA. ${ }^{7}$ Dept of Microbiology, New York University School of Medicine, New York, NY, USA.
\end{abstract}

Correspondence: Leopoldo N. Segal, NYU School of Medicine, 462 First Avenue 7W54, New York, NY 10016, USA. E-mail: Leopoldo.Segalanyumc.org

ABSTRACT Therapies targeting inflammation reveal inconsistent results in idiopathic pulmonary fibrosis (IPF). Aerosolised interferon (IFN) $-\gamma$ has been proposed as a novel therapy. Changes in the host airway microbiome are associated with the inflammatory milieu and may be associated with disease progression. Here, we evaluate whether treatment with aerosolised IFN- $\gamma$ in IPF impacts either the lower airway microbiome or the host immune phenotype.

Patients with IPF who enrolled in an aerosolised IFN- $\gamma$ trial underwent bronchoscopy at baseline and after 6 months. 16S rRNA sequencing of bronchoalveolar lavage fluid (BALF) was used to evaluate the lung microbiome. Biomarkers were measured by Luminex assay in plasma, BALF and BAL cell supernatant. The compPLS framework was used to evaluate associations between taxa and biomarkers.

IFN- $\gamma$ treatment did not change $\alpha$ or $\beta$ diversity of the lung microbiome and few taxonomic changes occurred. While none of the biomarkers changed in plasma, there was an increase in IFN- $\gamma$ and a decrease in Fit-3 ligand, IFN- $\alpha 2$ and interleukin-5 in BAL cell supernatant, and a decrease in tumour necrosis factor- $\beta$ in BALF. Multiple correlations between microbial taxa common to the oral mucosa and host inflammatory biomarkers were found.

These data suggest that the lung microbiome is independently associated with the host immune tone and may have a potential mechanistic role in IPF.

@ERSpublications

Lower airway microbiome and immunological tone are associated in IPF, an effect independent of IFN- $\gamma$ treatment http://ow.ly/cTDo30bsJiN

Cite this article as: Wang J, Lesko $\mathrm{M}$, Badri MH, et al. Lung microbiome and host immune tone in subjects with idiopathic pulmonary fibrosis treated with inhaled interferon- $\gamma$. ERJ Open Res 2017; 3 : 00008-2017 [https://doi.org/10.1183/23120541.00008-2017].

This article has supplementary material available from openres.ersjournals.com

Received: Jan 182017 | Accepted after revision: April 142017

This study is registered at www.clinicaltrials.gov with identifier number NCT00563212.

Support statement: This study was supported by US National Institutes of Health grant K23 AI102970 (to L.N. Segal). Funding information for this article has been deposited with the Crossref Funder Registry.

Conflict of interest: Disclosures can be found alongside this article at openres.ersjournals.com

Copyright $\odot$ ERS 2017. This article is open access and distributed under the terms of the Creative Commons Attribution Non-Commercial Licence 4.0. 


\section{Introduction}

Idiopathic pulmonary fibrosis (IPF) is a progressive, irreversible idiopathic interstitial lung disease with a median survival of 2-3 years. However, the rate of progression varies among individuals and is difficult to predict [1]. The evolving knowledge about the pathogenesis of this disease suggests that environmental factors cause repetitive injury to the alveolar epithelium followed by an abnormal repair process and scarring. The presence of comorbid conditions, such as emphysema and gastro-oesophageal reflux disease, may impact the lower airway microbiome and negatively affect prognosis in IPF [2-6]. With the increasing investigation of the lower airway microbiome using culture-independent techniques, observational studies have shown that in IPF there is increased bacterial burden and taxonomic differences [7, 8]. However, the role of the lower airway microbiome in the disease process is poorly understood.

Few therapies have been shown to change the natural history of IPF. Lung transplantation prolongs survival in patients with IPF but this option is limited primarily by the supply of donor organs. Post-transplant survival is poor in IPF when compared with other chronic lung diseases (e.g. cystic fibrosis). Pirfenidone (an anti-fibrotic and anti-inflammatory medication) and nintedanib (an oxindole derivative that inhibits signalling from platelet-derived growth factor (PDGF) receptor, vascular endothelial growth factor (VEGF) receptor and fibroblast growth factor (FGF) receptor) have been shown to slow the decline in forced vital capacity [1]. Interferon (IFN)- $\gamma$ is an endogenously produced T-helper type 1 (Th1) cytokine with anti-inflammatory, anti-proliferative, immunomodulatory and anti-fibrotic functions [9]. Although exogenous IFN- $\gamma$ was shown to be effective in vitro and in animal models of IPF [10-12], two randomised placebo controlled trials using subcutaneous $\operatorname{IFN}-\gamma[9,13]$ failed to demonstrate its therapeutic benefit in IPF patients. Inhaled IFN- $\gamma$ may be more effective than parenteral IFN- $\gamma$ [14]. We have conducted a phase II trial in subjects with IPF, and demonstrated that inhaled IFN- $\gamma$ can be safely delivered to lung parenchyma and that pulmonary function remained stable throughout the trial [15]. Using bronchoscopic samples obtained longitudinally in this pilot study, we explored possible mechanisms by which the lower airway microbiota interacts with the host. We evaluated associations between the lung microbiome and the local/systemic host immune phenotype during a clinical trial with aerosolised IFN- $\gamma$ in IPF patients.

\section{Methods}

Study design and participants

A prospective cohort study was designed to assess the efficacy of aerosolised IFN- $\gamma$ in patients with IPF. 10 patients between the ages of 40 and 70 years, diagnosed with IPF within the past year, were enrolled (see inclusion and exclusion criteria in the supplementary material). A baseline assessment was performed including physical exam, ECG, oxygen saturation by pulse oximetry and 6-min walk test (6MWT). Pulmonary function test (PFT) data from the previous 5 months and prior chest high-resolution computed tomography (HRCT) were reviewed. Baseline bronchoscopy was performed after patient consent. Inhaled IFN- $\gamma$ was delivered at a dose of $100 \mu \mathrm{g}$ via a nebuliser three times per week for a minimum of 80 weeks (supplementary figure S1). PFTs were obtained monthly, and repeat chest HRCT and bronchoscopy at 6 months. Data were stored in a secure database, which protected healthcare information. Bronchoscopy details and specimen handling are described in the supplementary material. The study was registered at ClinicalTrials.gov (NCT00563212).

\section{Quantitation and sequencing of bacterial 16S rRNA-encoding genes}

DNA was extracted from background and acellular BAL samples using an ion exchange column (Qiagen, Hilden, Germany). Library preparation and sequencing was performed on 16S rRNA gene amplicons encoding the V4 region on the Illumina MiSeq platform (Illumina, San Diego, CA, USA) as previously described $[16,17]$. The Shannon Index was calculated to evaluate $\alpha$ diversity (within sample diversity). Principal coordinate analysis was used to visualise $\beta$ diversity differences (between sample diversity). To compare $\alpha$, or taxonomy between groups at baseline, we used nonparametric (Mann-Whitney) tests. To evaluate changes in $\alpha$ diversity over time we utilised the Wilcoxon rank-sum test. $\beta$ diversity comparisons were based on PERMANOVA (permutational multivariate ANOVA).

\section{Measurement of biomarkers}

In vivo inflammation was assessed by BAL cell count, cell differential and measurement of biomarkers. 47 biomarkers were measured on a Luminex platform (Millipore, Billerica, MA, USA) in BALF, plasma and BAL cell supernatants (after $24 \mathrm{~h}$ of culture in RPMI medium). Measured levels of biomarkers were considered for analysis when data were above the lowest standard in $>50 \%$ of the samples (see supplementary material for further details). Biomarkers were categorised as Th1 (granulocyte-macrophage colony-stimulating factor (GM-CSF), IFN- $\gamma$, interleukin (IL)-2, IL-12 and tumour necrosis factor (TNF)- $\beta$ ), Th2 (IL-4, IL-5, IL-6, IL-9, IL-10, IL-13 and GM-CSF), Th17 (IL-1 $\beta$, IL-17, IL-6 and 
fractalkine) and IPF (IL-1 $\beta$, IL-17, IL-13, transforming growth factor (TGF)- $\beta$, monocyte chemoattractant protein (MCP)-1, IFN- $\gamma$, PDGF-AA and PDGF-AB-BB).

\section{Statistical analysis}

To avoid distributional assumptions for microbiome relative abundance data, we used nonparametric methods for univariate hypothesis testing. For microbiome associations with discrete factors, we used either the Mann-Whitney test (in the case of two categories) or the Kruskal-Wallis ANOVA (in the case of more than two categories). Paired statistics (Wilcoxon signed-rank test) were used for pre-post comparison of continuous covariates. For tests of association with continuous variables, we used nonparametric Spearman correlation tests. These tests were performed in $\mathrm{R}$ utilising the Hmisc package (https://cran.r-project.org). To evaluate differences between groups of $16 \mathrm{~S}$ data, we used the linear discriminant analysis (LDA) effect size (LEfSe) method [18]. Taxonomic features with an LDA score $>2.0$ were represented as a cladogram, as produced by LEfSe with default parameters. To adjust for statistically spurious associations, from compositional artefacts, multicollinearity and repeated measures design, we used the compPLS framework, outlined in RAMANAN et al. [19], to assess significant linear associations between genera relative abundances and biomarker measurements. On these networks we highlight profiles associated with Th1, Th2, Th17 and IPF immune responses [20]. Sequences are available from the National Center for Biotechnology Information Sequence Read Archive (www.ncbi.nlm.nih.gov/sra; accession SRP095188).

\section{Results}

\section{Cohort}

Subjects were enrolled and treated for this study from June 2008 until December 2009. Patients were enrolled within 4 weeks of screening. All 10 patients were followed for a minimum of 80 weeks with a range of $80-130$ weeks. All patients completed $>90 \%$ of scheduled visits. Three patients had a diagnosis confirmed by open lung biopsy reviewed by an independent pulmonary pathologist while the rest met clinical criteria with consistent chest HRCT scans. Histopathological diagnosis of confirmed usual interstitial pneumonia (UIP) was obtained in $40 \%$ of the subjects and pulmonary hypertension based on echo Doppler was found in $60 \%$ of the subjects. Table 1 presents demographic, clinical and PFT data.

$\begin{array}{lc}\text { TABLE } 1 \text { Demographics, pulmonary function and bronchoalveolar lavage (BAL) cell count } \\ \text { differential } \\ \text { Subjects } & 10 \\ \text { Age years } & 69.0(63.7-76.0) \\ \text { Male } & 8(80) \\ \text { Smoking status } & 0 \\ \quad \text { Current } & 5(50) \\ \text { Ex } & 5(50) \\ \text { Never } & 20(10-30) \\ \text { Pack-years (smokers) } & \\ \text { Clinical } & 4(40) \\ \text { Biopsy done } & 6(60) \\ \text { PASP elevated } & 36.8(33.2-40.7) \\ \text { PAP mmHg } & 4(40) \\ \mathbf{N} \text {-acetyl cysteine treatment } & \\ \text { Pulmonary function } & 73.5(68.2-84.2) \\ \text { FVC \% pred } & 71.0(60.0-77.0) \\ \text { TLC \% pred } & 47.5(39.5-56.5) \\ \text { DLCo \% pred } & 420(287-490) \\ \text { 6MWT m } & 19.2(13.8-31.9) \\ \text { BAL } & 78.0(69.0-91.2) \\ \text { BAL cells obtained } \times 10^{6} & 5.0(3.0-9.3) \\ \text { Macrophages \% } & 9.0(3.5-24.3) \\ \text { Lymphocytes \% } & 0.75(0-2.25) \\ \text { Neutrophils \% } & \\ \text { Eosinophils \% } & \\ & \end{array}$

Data are presented as $n$, median (interquartile range) or $n(\%)$. PASP: pulmonary artery systolic pressure; PAP: pulmonary arterial pressure; FVC: forced vital capacity; TLC: total lung capacity; DLCO: diffusing capacity of the lung for carbon monoxide; 6MWT: 6-min walk test. 
Median age was 69 years, $80 \%$ male and $50 \%$ ex-smokers. $40 \%$ of the patients received $\mathrm{N}$-acetyl cysteine treatment. None of the subjects received immunosuppressive therapy. PFTs showed a restrictive pattern with reduced mean (interquartile range) total lung capacity \% pred (71\% (60-77\%)), diffusing capacity of the lung for carbon monoxide \% pred (47.5\% (39.5-56.5\%)) and 6MWT (420 (287-490) m).

\section{Evaluation of the lung microbiome}

16S rRNA gene sequencing of BAL samples yielded a median (interquartile range) of 7611 (7017-9211) (lowest 4582) reads per sample. Technical negative controls yielded fewer reads (2959 (1628-4291); $\mathrm{p}<0.001)$ ). The three most abundant operational taxonomic units detected in sequences of technical negative controls were: Chitinophagaceae_550392, Betaproteobacteria_247441 and Nocardioidaceae_1088254 (relative abundance $12.3 \%$ (6.1-18.5\%), 12.3\% (5.1-18.4\%) and 11.9\% (5.9-17.9\%), respectively). None of these three taxa were found at $>0.01 \%$ in any given BAL sample. Figure 1 shows cluster analysis based on the most abundant taxa ( $>5 \%$ in any given sample) for all BAL samples. Consistent with prior observations, samples clustered in two groups: one enriched with genera commonly found in the upper airways, such as Prevotella, Streptococcus, Veillonella and Fusobacterium, and a second enriched with genera commonly described as background taxa (Pseudomonas, Acidocella and Flavobacterium) [21, 22]. To examine the effects of IFN treatment on the lung microbiome we evaluated changes in $\alpha$ and $\beta$ diversity. Figure $2 \mathrm{a}$ and $\mathrm{b}$ shows no significant difference in $\alpha$ or $\beta$ diversity between BAL samples at baseline and post IFN- $\gamma$ treatment. Evaluation of UniFrac distances within each subject (before versus after IFN- $\gamma$ treatment) and between different subjects shows a nonsignificant trend to a greater degree of similarity within subjects (figure 2c). However, a wide range of distances was noted within different subjects. This suggests that while in some subjects their lower airway microbiome remained resilient, in other subjects the microbiome underwent multiple changes in taxonomic composition. We then used LEfSe to evaluate taxonomic changes post IFN- $\gamma$ treatment. Figure $2 \mathrm{~d}$ shows a cladogram demonstrating very few significant taxonomic changes. Specifically, when compared with baseline, one genus annotated to the order Actinomycetales was enriched post IFN- $\gamma$ treatment, while only two genera annotated to the family Lachnospiraceae and order MIZ46 were decreased post IFN- $\gamma$ treatment. Notably, these changes occurred in genera with low relative abundance $(<1 \%)$.

\section{Evaluation of the host immune phenotype}

To evaluate the host immune phenotype, we measured BAL cell differentials and ex vivo biomarker production. Each BAL produced $>6.8 \times 10^{6}$ cells. Cell differential showed a predominance of alveolar macrophages $(78.0 \%(69.0-91.2 \%)$ ) with neutrophilia (>10\% of BAL cell differential) present in $50 \%$ of the samples (table 1). Supplementary table S1 shows biomarker levels in BALF, BAL cell ex vivo biomarker production, and levels in plasma at baseline and post IFN- $\gamma$ treatment. As expected, IFN- $\gamma$ concentration in BAL increased from undetectable levels to $294(161-1467) \mathrm{pg} \cdot \mathrm{mL}^{-1}$ post IFN- $\gamma$ treatment. In addition, IFN- $\gamma$ production by BAL cells ex vivo also increased from undetectable levels at baseline to 28 (15-46) $\mathrm{pg} \cdot \mathrm{mL}^{-1}$ in cell supernatants. Ex vivo BAL cell production of eotaxin, FGF-2, IFN- $\alpha 2$ and VEGF decreased, while there was a nonsignificant trend for a decreased production of IL- 8 , TNF- $\beta$ and PGDF-AA. Fewer changes were observed among plasma biomarkers. IL-7 had low levels at baseline and was undetectable post IFN- $\gamma$ treatment, while sIL-2Ra went from undetectable at baseline to low but measurable levels post IFN- $\gamma$ treatment.

\section{Correlation between microbiome and host immune phenotype}

We examined correlations between relative abundance of taxa and in vivo biomarker levels in plasma and BALF as well as ex vivo biomarker production of BAL cells. We found that taxa characteristic of upper airway microbiota, such as Rothia, Veillonella, Prevotella, Fusobacterium and Streptococcus, tend to coexist. We then determined that at baseline there were multiple positive and negative correlations identified between taxa and measured biomarkers in BALF, BAL cell supernatant and plasma (supplementary figure S2). We found fewer significant taxa-biomarker correlations among plasma biomarkers compared with BALF or BAL cell supernatant biomarkers. This suggests that the mucosal inflammatory phenotype is more tightly correlated with lower airway taxa than the systemic inflammatory phenotype. Most of the significant positive correlations occurred between biomarkers and taxa commonly considered upper airway commensals, such as Prevotella, Veillonella, Rothia and Streptococcus. Post IFN- $\gamma$ treatment, a different set of taxa were significantly correlated with levels of biomarkers (supplementary figure S2). However, similar to pre-treatment correlations, most of the significant positive correlations were still with taxa characteristic of upper airways.

We then used compPLS, which accounts for repeated measurements after IFN- $\gamma$ treatment to assess whether specific taxa were directly associated, after adjusting for fixed effects and multicollinearity, with biomarkers grouped within four immunological profiles: Th1, Th2, Th17 or IPF. Figure 3 shows that upper airway taxa were positively correlated with different biomarkers from each of these immunological 


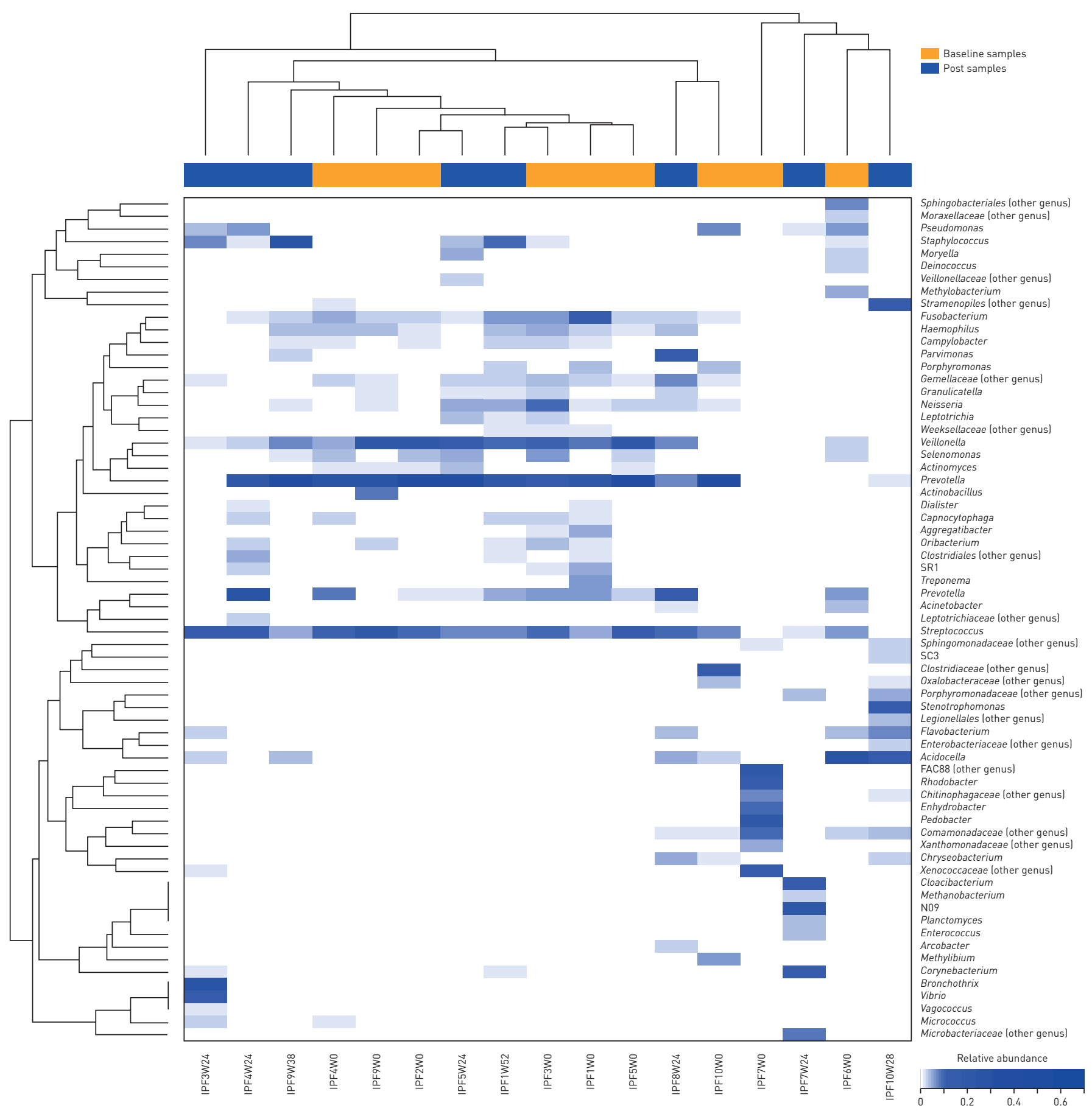

FIGURE 1 Unsupervised hierarchical clustering of most abundant taxa for bronchoalveolar lavage samples. The dendrogram demonstrates two well-separated clusters: one dominated by supraglottic taxa and a second one with a lower abundance of them. Clusters were not determined by pre- or post-treatment with interferon- $\gamma$.

profiles both at the local level (BALF and BAL cell supernatant) and the systemic level (plasma). For example, in BAL, Porphyromonas, an oral commensal, was positively correlated with levels of IL-1 $\beta$ and IL-6, two cytokines important for Th17 differentiation, and TNF- $\beta$, a cytokine characteristic of Th1 pathways. Dialister, another oral commensal, was positively correlated with levels of IL-4, IL-9 and IL-10, typical Th2 cytokines, as well as with IL- 2 and TNF- $\beta$, cytokines involved in Th1-mediated inflammation. Other cytokines with important roles previously described in IPF, such as MCP-1, IL-17, PDGF-AB-AA, were also correlated with taxa in BAL. For example, PDGF activates fibroblasts while IL-17 interacts with TGF- $\beta$ in pulmonary fibrosis [23]. Supplementary table S2 shows a summary of taxa associated with these 

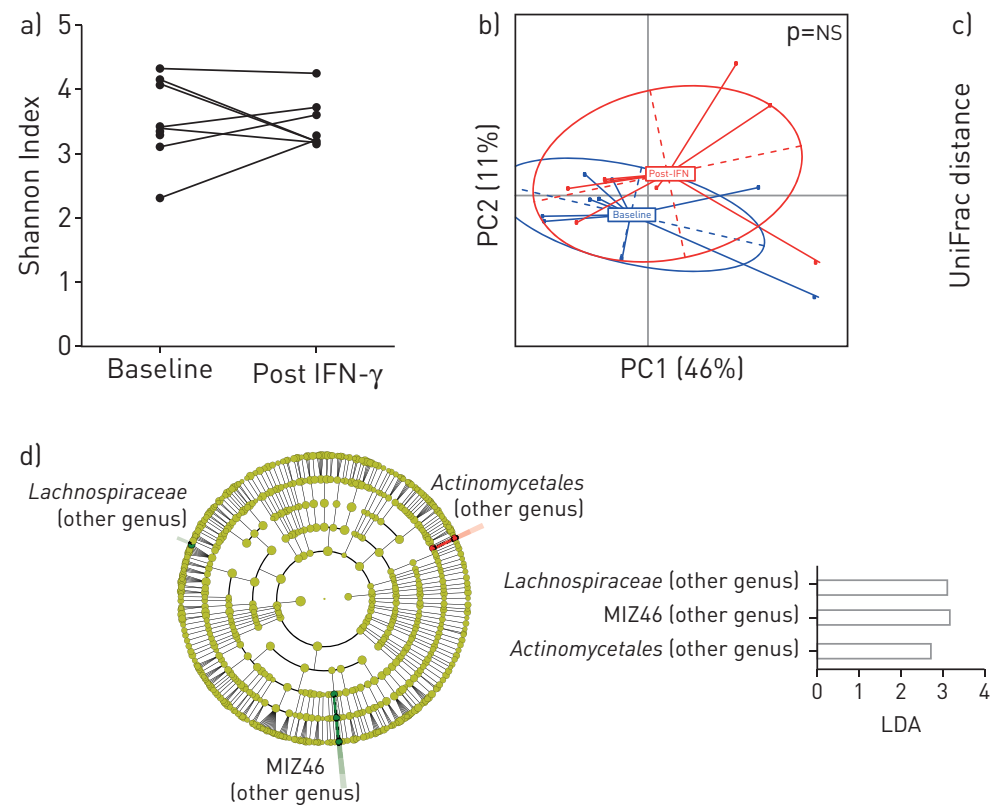
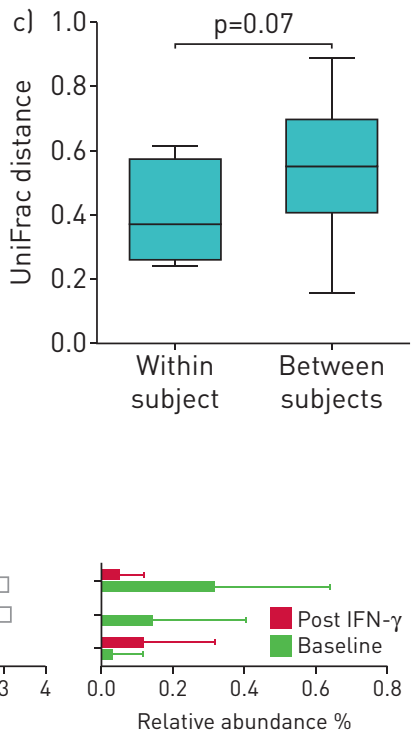

FIGURE 2 Microbiomic changes during inhaled interferon (IFN)- $\gamma$. a) Comparison of $\alpha$ diversity (based on the Shannon Index where sequences were rarified at 1000 reads per sample) before and after treatment. b) Changes in $\beta$ diversity (based on UniFrac distances) before and after IFN- $\gamma$ ( $p$-value based on PERMANOVA (permutational multivariate ANOVA)). PC: principal coordinate; NS: nonsignificant. c) $\beta$ diversity distance (based on UniFrac distances) between pairs of samples comparing within subject (before-after IFN- $\gamma$ ) versus between subjects ( $p$-value based on Mann-Whitney test). d) Linear discriminant analysis (LDA) effect size was calculated comparing $16 \mathrm{~S}$ rRNA data at baseline and after IFN- $\gamma$.

predefined immunological profiles (Th1, Th2, Th17 and IPF). The most abundant taxa identified were oral commensals, such as Prevotella, Parvimonas, Rothia and Porphyromonas. Taken together, the data support the interaction of oral commensals present in the lower airway with the host immune response.

\section{Discussion}

Our study sought to evaluate the effects of inhaled IFN- $\gamma$ on the lung microbiota, mucosa and systemic inflammatory phenotype of IPF patients. Consistent with previously published data, a large percentage of subjects with IPF had a lower airway microbiota enriched with oral microbes. Importantly, while IFN- $\gamma$ did not significantly change the lower airway microbiota, there was a correlation between oral microbes in the lower airways and the inflammatory phenotype, both local and systemic. The data support a tight interaction between the host and the lower airway microbiota in IPF.

IPF is a disease of lung injury and aberrant tissue repair where both innate and adaptive immunity play a role in the development of fibrosis [20]. The balance between the expression of Th1 and Th2 cytokines is an important determinant of disease progression in the inflammatory phase of pulmonary fibrosis [24]. Th1 biomarkers, such as IFN- $\gamma$, have been shown to ameliorate the degree of fibrosis in a bleomycin murine model, promoting its consideration as potential treatment in this disease [25]. However, Th2 biomarkers, such as, IL-5, have also been shown to potentiate fibrosis [26]. In our study, the IL-5 levels decreased in BAL cell supernatants as did some pro-inflammatory and pro-fibrotic biomarkers, such as IL-13, IL-17 and PDGF-AA. Interestingly, PDGF is a required element in fibroblast cellular division [23]. Activation of IL- 6 is associated with fibroblast proliferation. In our study, BAL and supernatant levels of IL-6 were decreased after treatment with aerosolised IFN- $\gamma$. Inhibition of IL- 6 has been associated with decreased pulmonary fibrosis in mouse models [27]. In contrast, no significant decreases in the levels of IL-13, IL-17, PDGF-AA and IL-6 were noted in systemic circulation from our subjects. These data suggest that inhaled IFN- $\gamma$ can alter multiple immunological and tissue repair pathways in the lower airway mucosa.

With recent advances in culture-independent techniques, there has been an increased awareness of the role of the lower airway microbiota in the host's immune response in multiple disease states [28]. In IPF, it is postulated that chronic microbial colonisation in the lower respiratory tract may contribute to the alveolar epithelial injury potentiating disease progression [29]. Microbes have been suspected of playing a role in the repetitive alveolar epithelial injury and aberrant wound healing that leads to the fibrotic process 

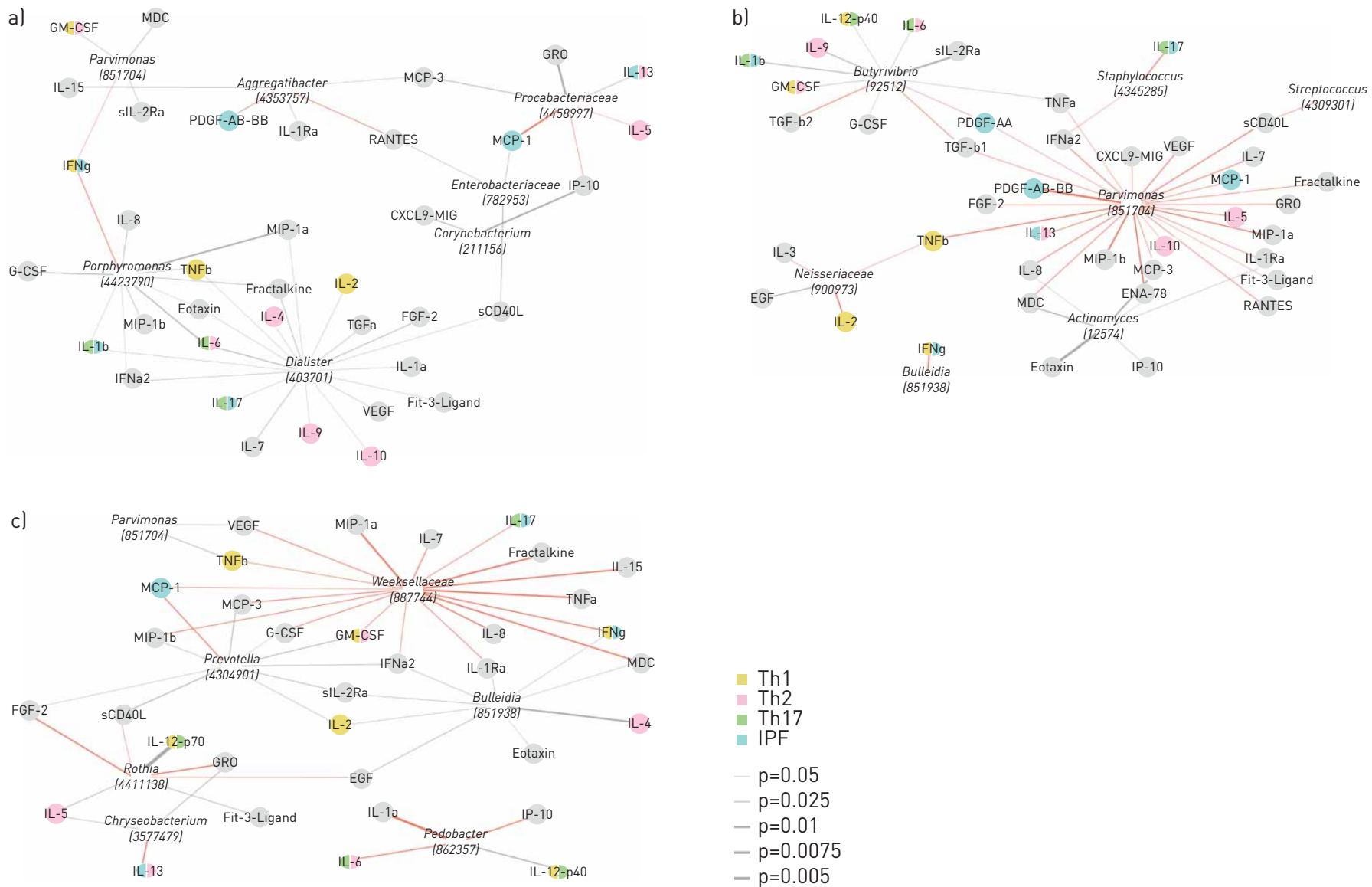

$$
\begin{aligned}
& \text { Th1 } \\
& \text { Th2 } \\
& \text { Th17 } \\
& \text { IPF } \\
& -p=0.05 \\
& -p=0.025 \\
& -p=0.01 \\
& -p=0.0075 \\
& -p=0.005
\end{aligned}
$$

FIGURE 3 Co-occurrence network between taxa and biomarkers. Th: T-helper; IPF: idiopathic pulmonary fibrosis. compPLS was utilised to evaluate associations between the lung microbiome and local/systemic biomarkers, which were categorised as Th1, Th2, Th17 and/or IPF immunological phenotype. a) Associations between taxa and bronchoalveolar lavage (BAL) fluid cytokines. b) Associations between taxa and BAL cell supernatant cytokines. c) Associations between taxa and plasma cytokines. Negative associations are in red and positive associations are in grey; edge weight is scaled to represent the degree of confidence for the associations.

characteristic of IPF. Thus, recent studies have begun to look at the potential role of bacterial pathogens in the development of fibrotic lung disease. Prior comparisons of BAL samples from subjects with IPF compared with subjects with normal lungs and chronic obstructive pulmonary disease (COPD) showed that in IPF there is an increase in bacterial burden in the lower airways [7]. Furthermore, there is a direct correlation between this lower airway bacterial burden and the decline in lung function. In a large multicentre trial, Staphylococcus and Streptococcus were associated with progression of disease in IPF [8]. In a double-blind randomised placebo controlled trial, cotrimoxazole was given to patients with IPF twice daily for a period of 12 months, leading to improvement in quality of life scoring and decreased mortality [29]. In our study, the diversity of the lower airway microbiota of patients with IPF did not significantly change during treatment with aerosolised IFN- $\gamma$. However, taxonomic composition of the lower airway microbiota was significantly associated with multiple immunological pathways that may play a role in the lung parenchymal injury/repair process in IPF. In healthy smokers, we have shown that a lower airway microbiome enriched with taxa commonly found in the upper airways is associated with a distinct immune profile characterised by a Th17 phenotype and a blunted Toll-like receptor 4 response [16]. We have also noted that this distinct lung microbiome (pneumotype SPT $_{\text {for "supraglottic predominant taxa") }}$ is associated with a distinct genomic potential and metabolomic profile. In line with our prior investigations, we show that in IPF, taxa commonly characterised as oral commensals are present in the lower airways and are associated with pre-specified immunological profiles (Th1, Th2, Th17 and IPF). Furthermore, a recent investigation showed that a disrupted lower airway microbiota was associated with host transcriptome differences and this association remained significant during longitudinal follow-up [30]. Although the causative nature of this association is beyond the scope of our study, data suggest that the lung microbiota may represent an important environmental factor in the pathogenesis of this disease by either promoting lung injury or altering the repair process of the injured lung tissue. The exact mechanism for this association still needs to be elucidated with a focus on microbial metabolites and 
host-microbe interactions. In subjects with early COPD, we have shown that microbial metabolism is a potential anti-inflammatory therapeutic target [31], raising the possibility that it may also be a potential therapeutic target to prevent further lung tissue damage in IPF.

There are several limitations in our study. In this IPF cohort, the vast majority of the subjects were diagnosed with HRCT and without biopsy. Conversely, in a similar clinical setting, HRCT has been found to have a $90 \%$ positive predictive value for the histopathological diagnosis of UIP [32]. Although we followed patients for a minimum of 80 weeks, the sample size is small with only 10 patients enrolled. However, this was a pilot study primarily designed to evaluate the feasibility and safety of treatment with inhaled IFN- $\gamma$ that required two bronchoscopies. There are few longitudinal studies that have evaluated the lower airway microbiota and none that we are aware of in IPF. Future studies will require a larger cohort and the use of noninvasive methods to evaluate the lower airway microbiota and biomarkers. Additionally, since we lack a placebo control group, the observed changes in microbiome and biomarkers need to be interpreted with caution. Sampling with bronchoscopy was restricted to only one site and spatial variation (especially since IPF preferentially affects the lower lung parenchyma) could not be investigated [33]. We could not assess the upper airway microbiota as we did not concomitantly collect oral samples. Thus, we cannot control for cross-contamination with oral secretions. Although contamination with oropharyngeal microbiota can occur during bronchoscopy, recent data suggest that sampling artefacts of the lower airway microbial surveys are relatively minor [21,33-35]. In this investigation, we utilised acellular BAL to characterise the lower airway microbiota. Therefore, it is possible that cell-associated microbes are underrepresented [36], further affecting the degree of background enrichment due to decreased of lower airway bacterial load. Moreover, although we included technical controls in sequencing runs, BAL samples were obtained prior to adapting our protocol for sampling bronchoscopic environmental controls, which may also be important for microbiome studies with low biomass samples [22, 37].

In summary, inhaled IFN- $\gamma$ was associated with small changes in the composition of the lower airway microbiota. Importantly, multiple correlations were found between the microbiome and inflammatory and fibrotic biomarkers in the lung mucosa. These associations were maintained when considering IFN- $\gamma$ treatment in a multiomic model. Taken together, these data suggest that the lower airway microbiome is independently associated with the host immune tone and thus may provide a novel therapeutic target for treatment of IPF.

\section{Acknowledgements}

Author contributions: Conception and design: G. Smaldone, R. Condos, L.N. Segal. Acquisition of data: Y. Li, L.N. Segal. Analysis and interpretation of data: J. Wang, M. Lesko, M.H. Badri, B. Kapoor, B.G. Wu, Y. Li, R. Bonneau, Z.D. Kurtz, L.N. Segal. Drafting or revising of article: J. Wang, M. Lesko, M.H. Badri, B.G. Wu, Z.D. Kurtz, R. Condos, L.N. Segal. Final approval of the manuscript: J. Wang, M. Lesko, M.H. Badri, B. Kapoor, Y. Li, G. Smaldone, Z.D. Kurtz, R. Condos, L.N. Segal.

\section{References}

1 Spagnolo P, Sverzellati N, Rossi G, et al. Idiopathic pulmonary fibrosis: an update. Ann Med 2015; 47: 15-27.

2 Raghu G, Freudenberger TD, Yang S, et al. High prevalence of abnormal acid gastro-oesophageal reflux in idiopathic pulmonary fibrosis. Eur Respir J 2006; 27: 136-142.

3 Salvioli B, Belmonte G, Stanghellini V, et al. Gastro-oesophageal reflux and interstitial lung disease. Dig Liver Dis 2006; 38: 879-884.

4 Tobin RW, Pope CE 2nd, Pellegrini CA, et al. Increased prevalence of gastroesophageal reflux in patients with idiopathic pulmonary fibrosis. Am J Respir Crit Care Med 1998; 158: 1804-1808.

5 Cottin V, Cordier JF. Combined pulmonary fibrosis and emphysema: an experimental and clinically relevant phenotype. Am J Respir Crit Care Med 2005; 172: 1605.

6 Cottin V, Nunes H, Brillet PY, et al. Combined pulmonary fibrosis and emphysema: a distinct underrecognised entity. Eur Respir J 2005; 26: 586-593.

7 Molyneaux PL, Cox MJ, Willis-Owen SA, et al. The role of bacteria in the pathogenesis and progression of idiopathic pulmonary fibrosis. Am J Respir Crit Care Med 2014; 190: 906-913.

8 Han MK, Zhou Y, Murray S, et al. Lung microbiome and disease progression in idiopathic pulmonary fibrosis: an analysis of the COMET study. Lancet Respir Med 2014; 2: 548-556.

9 Raghu G, Brown KK, Bradford WZ, et al. A placebo-controlled trial of interferon gamma-1b in patients with idiopathic pulmonary fibrosis. $N$ Engl J Med 2004; 350: 125-133.

10 Narayanan AS, Whithey J, Souza A, et al. Effect of gamma-interferon on collagen synthesis by normal and fibrotic human lung fibroblasts. Chest 1992; 101: 1326-1331.

11 Okada T, Sugie I, Aisaka K. Effects of gamma-interferon on collagen and histamine content in bleomycin-induced lung fibrosis in rats. Lymphokine Cytokine Res 1993; 12: 87-91.

12 Clark JG, Dedon TF, Wayner EA, et al. Effects of interferon-gamma on expression of cell surface receptors for collagen and deposition of newly synthesized collagen by cultured human lung fibroblasts. J Clin Invest 1989; 83: $1505-1511$.

13 King TEJr, Albera C, Bradford WZ, et al. Effect of interferon gamma-1b on survival in patients with idiopathic pulmonary fibrosis (INSPIRE): a multicentre, randomised, placebo-controlled trial. Lancet 2009; 374: 222-228.

14 Dawson R, Condos R, Tse D, et al. Immunomodulation with recombinant interferon-gammalb in pulmonary tuberculosis. PLoS One 2009; 4: e6984. 
15 Diaz KT, Skaria S, Harris K, et al. Delivery and safety of inhaled interferon-gamma in idiopathic pulmonary fibrosis. J Aerosol Med Pulm Drug Deliv 2012; 25: 79-87.

16 Segal LN, Clemente JC, Tsay JC, et al. Enrichment of the lung microbiome with oral taxa is associated with lung inflammation of a Th17 phenotype. Nat Microbiol 2016; 1: 16031.

17 Scher JU, Joshua V, Artacho A, et al. The lung microbiota in early rheumatoid arthritis and autoimmunity. Microbiome 2016; 4: 60 .

18 Segata N, Izard J, Waldron L, et al. Metagenomic biomarker discovery and explanation. Genome Biol 2011; 12: R60.

19 Ramanan D, Bowcutt R, Lee SC, et al. Helminth infection promotes colonization resistance via type 2 immunity. Science 2016; 352: 608-612.

20 Kolahian S, Fernandez IE, Eickelberg O, et al. Immune mechanisms in pulmonary fibrosis. Am J Respir Cell Mol Biol 2016; 55: 309-322.

21 Segal LN, Alekseyenko AV, Clemente JC, et al. Enrichment of lung microbiome with supraglottic taxa is associated with increased pulmonary inflammation. Microbiome 2013; 1: 19.

22 Salter SJ, Cox MJ, Turek EM, et al. Reagent and laboratory contamination can critically impact sequence-based microbiome analyses. BMC Biol 2014; $12: 87$.

23 Alvarez RH, Kantarjian HM, Cortes JE. Biology of platelet-derived growth factor and its involvement in disease. Mayo Clin Proc 2006; 81: 1241-1257.

24 Wynn TA. Integrating mechanisms of pulmonary fibrosis. J Exp Med 2011; 208: 1339-1350.

25 Keane MP, Belperio JA, Burdick MD, et al. IL-12 attenuates bleomycin-induced pulmonary fibrosis. Am J Physiol Lung Cell Mol Physiol 2001; 281: L92-L97.

26 Wynn TA. Fibrotic disease and the $\mathrm{T}_{\mathrm{H}} 1 / \mathrm{T}_{\mathrm{H}} 2$ paradigm. Nat Rev Immunol 2004; 4: 583-594.

27 Le TT, Karmouty-Quintana H, Melicoff E, et al. Blockade of IL-6 trans signaling attenuates pulmonary fibrosis. $J$ Immunol 2014; 193: 3755-3768.

28 Segal LN, Rom WN, Weiden MD. Lung microbiome for clinicians. New discoveries about bugs in healthy and diseased lungs. Ann Am Thorac Soc 2014; 11: 108-116.

29 Shulgina L, Cahn AP, Chilvers ER, et al. Treating idiopathic pulmonary fibrosis with the addition of co-trimoxazole: a randomised controlled trial. Thorax 2013; 68: 155-162.

30 Molyneaux PL, Willis-Owen SAG, Cox MJ, et al. Host-microbial interactions in idiopathic pulmonary fibrosis. Am J Respir Crit Care Med 2017; 195: 1640-1650.

31 Segal LN, Clemente JC, Wu BG, et al. Randomised, double-blind, placebo-controlled trial with azithromycin selects for anti-inflammatory microbial metabolites in the emphysematous lung. Thorax 2017; 72: 13-22.

32 Sgalla G, Biffi A, Richeldi L. Idiopathic pulmonary fibrosis: diagnosis, epidemiology and natural history. Respirology 2016; 21: 427-437.

33 Dickson RP, Erb-Downward JR, Freeman CM, et al. Bacterial topography of the healthy human lower respiratory tract. mBio 2017; 8: e02287-16.

34 Bassis CM, Erb-Downward JR, Dickson RP, et al. Analysis of the upper respiratory tract microbiotas as the source of the lung and gastric microbiotas in healthy individuals. mBio 2015; 6: e00037-15.

35 Dickson RP, Erb-Downward JR, Martinez FJ, et al. The microbiome and the respiratory tract. Annu Rev Physiol 2016; 78: 481-504.

36 Dickson RP, Erb-Downward JR, Prescott HC, et al. Cell-associated bacteria in the human lung microbiome. Microbiome 2014; 2: 28.

37 Segal LN, Dickson RP. The lung microbiome in HIV. Getting to the HAART of the host-microbe interface. Am J Respir Crit Care Med 2016; 194: 136-137. 